\title{
Diagnosticando o Transtorno Autista: Aspectos Fundamentais e Considerações Práticas
}

Diagnosing Autistic Disorder:

Fundamental Aspects and Practical Considerations

Diagnosticando el Trastorno Autista: Aspectos Fundamentales y Consideraciones Prácticas

Micheline Silva Universidade de Brasília

James A. Mulick Autism Center \& Developmental Assessment Program Columbus, Ohio, EUA
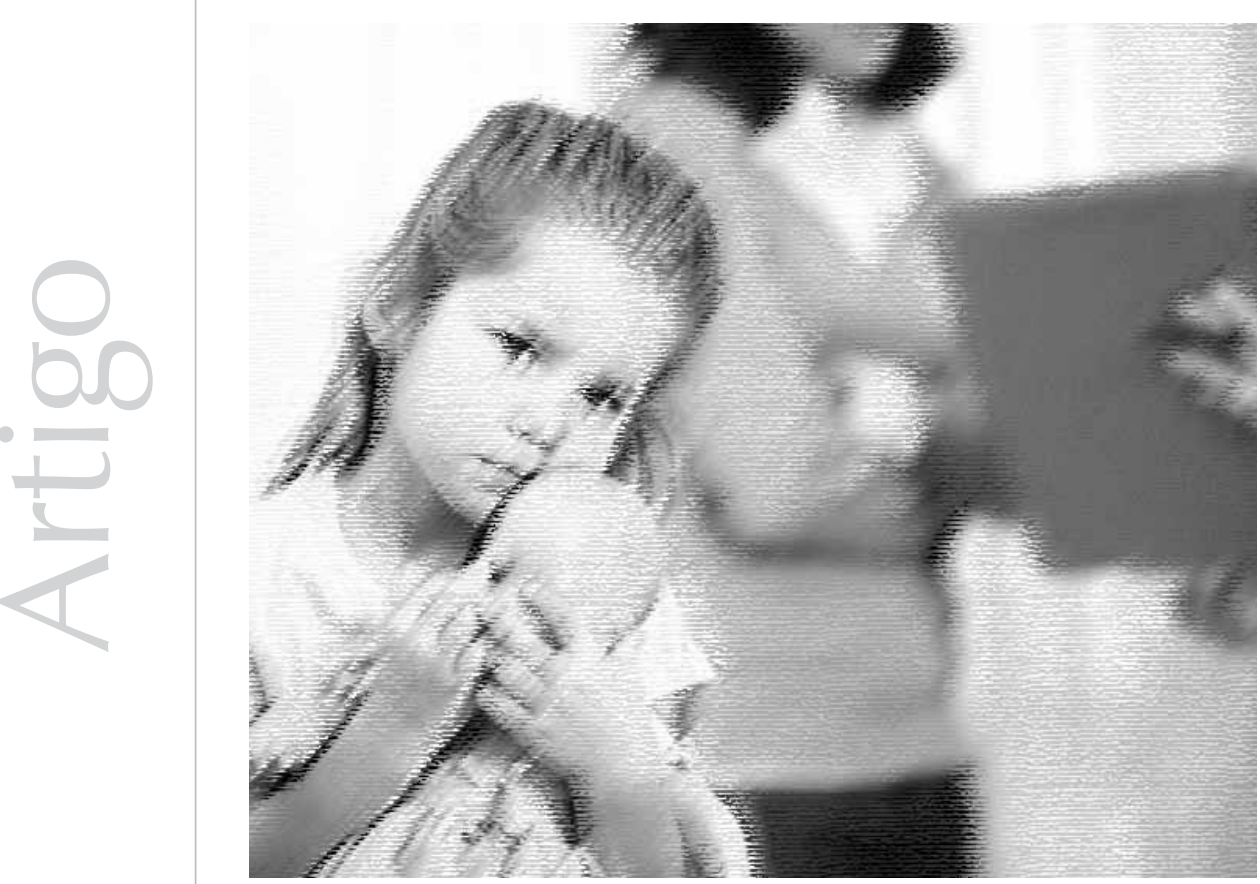
Resumo: Tendo como base modelos de prática diagnóstica implementados em outros países, o objetivo do artigo é oferecer uma revisão geral acerca do que vem a ser o transtorno autista e dos fatores críticos que devem ser considerados durante o processo diagnóstico. São discutidos aspectos dos critérios diagnósticos e também das comorbidades, incidência, etiologia e diretrizes para a prática diagnóstica. Tais diretrizes incluem formas de exploração de sintomas de risco durante exames de rotina realizados por profissionais que trabalham com a população infantil e elementos básicos necessários para a realização de uma avaliação minuciosa e criteriosa por uma equipe interdisciplinar. Isso inclui, por exemplo, o uso de instrumentos específicos auxiliares no diagnóstico, elementos importantes para a avaliação médica e psicológica e encaminhamentos para serviços adequados de intervenção e apoio.

Palavras-chave: Transtorno autista. Diagnóstico precoce. Prática diagnóstica. Equipe interdisciplinar.

Abstract: Based on diagnostic models implemented in other countries, the goal of the article is to offer a general overview about autistic disorder and highlight some critical elements to be taken into account during the diagnostic process. We discuss aspects regarding the diagnostic criteria, as well as regarding comorbidities, incidence, etiology, and some practical guidelines for determining a diagnosis. Such guidelines include the critical aspects to be considered when screening the risk for autism in early childhood population and for a careful and comprehensive evaluation by a specialized interdisciplinary team. A comprehensive evaluation consists of the use of specific tools to help determine the diagnosis, some crucial elements for the medical and psychological evaluation, and the referral to adequate intervention and support services. Keywords: Autism disorder. Early diagnosis. Diagnostic practice. Interdisciplinary team.

Resumen: Teniendo como base modelos de práctica diagnóstica implementados en otros países, el objetivo del artículo es ofrecer una revisión general acerca de lo que viene a ser el trastorno autista y de los factores críticos que deben ser considerados durante el proceso diagnóstico. Son discutidos aspectos de los criterios diagnósticos y también de las comorbidades, incidencia, etiología y directrices para la práctica diagnóstica. Tales directrices incluyen formas de exploración de síntomas de riesgo durante exámenes de rutina realizados por profesionales que trabajan con la población infantil y elementos básicos necesarios para la realización de una evaluación minuciosa y de criterio por un equipo interdisciplinario. Eso incluye, por ejemplo, el uso de instrumentos específicos auxiliares en el diagnóstico, elementos importantes para la evaluación médica y psicológica y encaminamientos para servicios adecuados de intervención y apoyo.

Palabras clave: Trastorno autista. Diagnóstico precoz. Práctica diagnóstica. Equipo interdisciplinario.

O objetivo principal do artigo é oferecer uma revisão geral acerca do que vem a ser o transtorno autista e ressaltar os fatores críticos que devem ser considerados durante o processo diagnóstico. Vale salientar que o conteúdo aqui apresentado se baseia em práticas que já vêm sendo, por algum tempo, implementadas em outros países, mas que ainda não se apresentam como uma realidade bem consolidada no Brasil. Assim, esperamos que as diretrizes aqui apontadas possam instigar reflexões acerca das atuais práticas diagnósticas no Brasil, auxiliando no desenvolvimento de estratégias mais eficazes de diagnóstico e de sistemas de apoio mais adequados.
O transtorno autista (ou autismo infantil) faz parte de um grupo de transtornos do neurodesenvolvimento denominados Transtornos Globais do Desenvolvimento (TGDs), Transtornos Invasivos do Desenvolvimento (TIDs) ou Transtornos do Espectro do Autismo (TEAs). Esse grupo de transtornos compartilha sintomas centrais no comprometimento em três áreas específicas do desenvolvimento, a saber: (a) déficits de habilidades sociais, (b) déficits de habilidades comunicativas (verbais e não-verbais) e (c) presença de comportamentos, interesses e/ou atividades restritos, repetitivos e estereotipados. Além do transtorno autista, fazem parte desse grupo 
Em vários países, como nos

Estados Unidos, a média de idade das crianças

diagnosticadas tem sido de 3 a 4 anos (Chakrabarti \& Fombonne, 2001, 2005;

Charman \& Baird, 2002; Filipek et al., 1999), mas, mesmo assim, muitos pais já começam a notar que existe algum problema com a sua criança antes do segundo ano de vida e, em alguns casos, até no primeiro ano de vida da criança

(Charman \& Baird, 2002). o transtorno (ou síndrome) de Asperger, o transtorno desintegrativo da infância (ou outro transtorno desintegrativo da infância), o transtorno (ou síndrome) de Rett e o transtorno global do desenvolvimento - sem outra especificação (incluindo o autismo atípico) (World Health Organization [WHO], 1992; American Psychiatric Association [APA], 2003).

A incidência de casos de autismo tem crescido de forma significativa em todo o mundo, especialmente durante as últimas décadas (Barbaresi, Katusic, \& Voigt, 2006; Fombonne, Zakarian, Bennett, Meng, \& Mclean-Heywood, 2006; Gernsbacher, Dawson, \& Goldsmith, 2005; Schechter \& Grether, 2008). Desse modo, profissionais da saúde, educação e áreas afins, que tenham a infância como especialidade, devem estar cada vez mais preparados para se deparar com casos de autismo nas suas práticas. Entretanto, ainda hoje existe uma grande lacuna em termos de conhecimento e capacitação profissional em relação às práticas diagnósticas e à implementação de programas de intervenção. Apesar de ter havido enormes avanços nessas últimas décadas em relação à identificação precoce e ao diagnóstico de autismo, muitas crianças, especialmente no Brasil, ainda continuam por muitos anos sem um diagnóstico ou com diagnósticos inadequados.

Em vários países, como nos Estados Unidos, a média de idade das crianças diagnosticadas tem sido de 3 a 4 anos (Chakrabarti \& Fombonne, 2001, 2005; Charman \& Baird, 2002; Filipek et al., 1999), mas, mesmo assim, muitos pais já começam a notar que existe algum problema com a sua criança antes do segundo ano de vida e, em alguns casos, até no primeiro ano de vida da criança (Charman \& Baird, 2002). Desse modo, tem se tornado cada vez mais possível diagnosticar crianças com idades entre 18 e 24 meses
(Goldberg et al., 2003; Lord, Shulman, \& Dilavore, 2004; Siperstein \& Volkmar, 2004; Werner \& Dawson, 2005) ou até mesmo mais novas - entre 6 e 12 meses (Charman \& Baird, 2002; Filipek et al., 1999; Zwaigenbaum et al., 2005). No Brasil, por outro lado, nossa experiência prática tem indicado que, em vários Estados, muitas crianças ainda permanecem com um diagnóstico em aberto até as idades de 6 ou 7 anos e até mesmo por mais tempo. Assim, faz-se necessário que modelos de prática diagnóstica que têm se mostrado eficientes e adequados em outros países (como o que será aqui apresentado) bem como em alguns Estados do Brasil (como, por exemplo, em São Paulo - e.g., Mercadante, Gaag, \& Schwartzman, 2006), se tornem cada vez mais parte das discussões entre os profissionais da área, servindo como diretrizes para o aprimoramento das práticas diagnósticas implementadas no Brasil como um todo.

\section{Critérios diagnósticos}

O diagnóstico de autismo é estabelecido com base em uma lista de critérios comportamentais. Em vários países da Europa e da América do Norte, incluindo os Estados Unidos e o Canadá, especialistas na área recomendam que o diagnóstico seja feito com base nos critérios estabelecidos pelo ICD-10 (WHO, 1992) e/ou pelo DSM-IV-TR (APA, 2003). No presente artigo, focalizaremos os critérios diagnósticos oferecidos pelo DSMIV-TR (APA, 2003).

Segundo os critérios do DSM-IV-TR, para que a criança seja diagnosticada com transtorno autista, ela deve apresentar pelo menos seis da lista de doze sintomas apresentados na Tabela 1 , sendo que pelo menos dois dos sintomas devem ser na área de interação social, pelo menos um na área de comunicação, e pelo menos um na área de comportamentos restritos, repetitivos e estereotipados. 
Tabela 1. Lista de sintomas do transtorno autista, por área, de acordo com os critérios oferecidos pelo DSM-IV-TR (APA, 2003)

Comprometimento qualitativo da interação social:

(a) Comprometimento acentuado no uso de múltiplos comportamentos não-verbais, tais como contato visual direto, expressão facial, posturas corporais e gestos para regular a interação social;

(b) Fracasso em desenvolver relacionamentos com seus pares apropriados ao nível de desenvolvimento (i.e., à sua faixa etária);

(c) Ausência de tentativas espontâneas de compartilhar prazer, interesses ou realizações com outras pessoas (ex., não mostrar, trazer ou apontar objetos de interesse);

Ausência de reciprocidade social ou emocional.

Comprometimento qualitativo da comunicação:

(a) Atraso ou ausência total de desenvolvimento da linguagem falada (não acompanhado por uma tentativa de compensar por meio de modos alternativos de comunicação, tais como gestos ou mímica);

(b) Em indivíduos com fala adequada, acentuado comprometimento da capacidade de iniciar ou manter uma conversa;

(c) Uso estereotipado e repetitivo da linguagem ou linguagem idiossincrática;

(d) Ausência de jogos ou brincadeiras de imitação social variados e espontâneos próprios do nível de desenvolvimento (i.e., da sua faixa etária).

Padrões restritos e repetitivos de comportamento, interesses e atividades:

(a) Preocupação insistente com um ou mais padrões estereotipados e restritos de interesse, anormais em intensidade ou foco;

(b) Adesão aparentemente inflexível a rotinas ou rituais específicos e não-funcionais;

(c) Maneirismos motores estereotipados e repetitivos (ex., agitar ou torcer mãos e dedos ou movimentos complexos de todo o corpo);

(d) Preocupação persistente com partes de objetos.

Além disso, a criança deve também ter começado a exibir atrasos (ou funcionamento atípico), até a idade de três anos, em, pelo menos, uma das seguintes áreas: (1) interação social, (2) linguagem para fins de comunicação social ou (3) brincadeiras ou jogos simbólicos ou imaginários. Vale ressaltar, ainda, que o diagnóstico de transtorno autista apenas deve ser estabelecido quando o quadro não for mais bem explicado pelo transtorno de Rett ou pelo transtorno desintegrativo da infância, que não estão sendo aqui discutidos, mas que fazem parte dos transtornos globais do desenvolvimento (TGDs) não-autísticos (Mercadante et al., 2006).

\section{Comorbidades e alterações associadas}

\section{Outras condições e transtornos}

Além de um diagnóstico diferencial, faz-se necessária também a identificação de condições que coexistam com um quadro de autismo. A condição que mais comumente coexiste com o autismo é o retardo mental, presente em níveis de severidade variados em aproximadamente 60 a 75\% das crianças com autismo (e.g., Bailey, Philips, \& Rutter, 1996; Barbaresi, Katusic, Colligan, Weaver, \& Jacobsen, 2005). 
Existem também casos nos quais quadros de autismo foram identificados como coexistentes em indivíduos com outros transtornos, como síndrome de Down, paralisia cerebral e síndrome de Tourette, bem como deficiências visuais e auditivas (Charman \& Baird, 2002). Quadros de transtornos depressivos e de ansiedade são também comuns em adolescentes e adultos com autismo de alto funcionamento cognitivo (Newsom \& Hovanitz, 2006).

\section{Problemas comportamentais}

Crianças com autismo freqüentemente apresentam problemas de comportamento, muitas vezes bastante severos, que incluem hiperatividade, dificuldade de prestar e/ou manter atenção, atenção hiperseletiva (i.e., tendência a prestar mais atenção nas partes/ detalhes do que no todo) e impulsividade, bem como comportamentos agressivos, autodestrutivos, perturbadores e destrutivos. Especialmente em crianças mais novas, comumente se observa uma baixa tolerância à frustração, acompanhada por "acessos de raiva" e "escândalos" - jogar-se no chão, gritar, chorar, bater com a cabeça, se morder, bater nos outros etc. (e.g., Barbaresi et al., 2005; Lindsay \& Aman, 2003; Newsom \& Hovanitz, 2006).

\section{Problemas sensoriais}

Também é bastante comum se observar em crianças autistas respostas sensoriais e perceptuais peculiares, incluindo hiper ou hiposensibilidade a estímulos sonoros, visuais, táteis, olfativos e gustativos, além de alto limiar para a dor física e um medo exagerado de estímulos ordinariamente considerados inofensivos. Assim, é comum se observar crianças autistas cobrindo os ouvidos e chorando ao ouvir sons triviais, como sons de uma descarga de banheiro ou de pessoas falando alto, ou, ao contrário, não emitindo qualquer resposta observável a estímulos sonoros em alto volume, como alguém batendo em uma panela ao seu lado. É também comum se observar crianças autistas fascinadas por certos estímulos visuais, como luzes piscando e reflexos de espelho bem como tendo certas aversões ou preferências por gostos, cheiros e texturas específicas - como se recusar a tocar certas texturas, ou ficar fascinado com certas texturas tocando, lambendo ou mesmo comendo indiscriminadamente coisas que apresentem tal textura, mesmo que não sejam comestíveis etc. (e.g., Charman \& Baird, 2002; Filipek et al., 1999; Newsom \& Hovanitz, 2006).

\section{Problemas médicos}

Problemas gastrointestinais são freqüentemente observados em crianças autistas, incluindo episódios recorrentes de diarréia e/ou constipação, além de refluxo, alergias ou intolerâncias alimentares (e.g., Newsom \& Hovanitz, 2006). Crianças autistas também tendem a apresentar problemas severos de alimentação e de sono (e.g., Nash \& Coury, 2003). Quanto à alimentação, é comum, por exemplo, casos de dieta hiperseletiva, que, muitas vezes, levam a problemas de saúde como desnutrição, além de casos de Pica (e.g., Filipek et al., 1999), condição na qual há um consumo persistente de substâncias não nutritivas e não comestíveis, que freqüentemente pode levar a intoxicações, como a intoxicação por chumbo (e.g., Shannon \& Graef, 1997). Em relação ao sono, observa-se dificuldade para adormecer bem como para se manter adormecido durante todo o ciclo de sono, entre outros (e.g., Schreck \& Mulick, 2000; Newsom \& Hovanitz, 2006).

Muitas crianças autistas também apresentam uma história de otite recorrente e, em alguns 
casos, de problemas auditivos (e.g., Barbaresi et al., 2006; Newsom \& Hovanitz, 2006). Problemas neurológicos podem também estar presentes. Em alguns casos, indivíduos autistas podem vir a desenvolver transtornos convulsivos ou epilepsia durante a infância ou adolescência (e.g., Barbaresi et al., 2006; Filipek et al., 1999; Nash \& Coury, 2003; Newsom \& Hovanitz, 2006). Mesmo na ausência de convulsões ou epilepsia, em muitos casos, estudos de imagens e de atividades cerebrais revelam irregularidades neurológicas, sem que seja possível identificar padrões específicos de anormalidade (e.g., Barbaresi et al., 2006; Charman \& Baird, 2002; Filipek et al., 1999; Newsom \& Hovanitz, 2006).

\section{Etiologia}

Existe certo consenso entre os especialistas de que o autismo é decorrente de disfunções do sistema nervoso central (SNC), que levam a uma desordem no padrão do desenvolvimento da criança. Estudos de neuroimagens e de autópsias, por exemplo, apontam uma variedade de anormalidades cerebrais em indivíduos com autismo, como tamanhos anormais das amígdalas, hipocampos e corpo caloso, maturação atrasada do córtex frontal, desenvolvimento atrofiado dos neurônios do sistema límbico e padrões variados de baixa atividade em regiões cerebrais diversas, como o córtex frontal e o sistema límbico (e.g., Brambilla et al., 2003; Muller, Kleinhans, Kemmotsu, Pierce, \& Courchesne, 2003; Mundy, 2003; Redcay \& Courchesne, 2005). Além disso, estudos têm também evidenciado anormalidades no padrão de crescimento cerebral de crianças autistas, que, ao nascer, tendem a exibir uma circunferência craniana menor que a média, mas, entre as idades de um e dois meses e de seis e quatorze meses, apresentam um crescimento acelerado e excessivo da cabeça, mesmo em comparação a crianças com transtorno invasivo do desenvolvimento - sem outra especificação (e.g., Courchesne, Caper, \& Akshoomoff, 2003; Redcay \& Courchesne, 2005).

Apesar dessas evidências de anormalidades neurodesenvolvimentais vinculadas ao autismo, por conta da complexidade do SNC, bem como da grande variabilidade de manifestações sintomatológicas, até o presente momento, não foi possível determinar qualquer aspecto biológico, ambiental, ou da interação de ambos, que pareça contribuir de forma decisiva para a manifestação desse transtorno. Devido ao fato de o autismo se manifestar bastante cedo durante o desenvolvimento da criança, um grande esforço tem sido direcionado para a exploração de anomalias genéticas ou para a exposição a eventos ambientais específicos no início da vida como possivelmente relacionados à emergência de tal desordem. Porém, até recentemente, apenas 3 a 5\% dos casos de autismo é estimado como vinculado a algum tipo de condição médica (Challman, Barbaresi, Katusic, \& Weaver, 2003). Nesses casos, geralmente também se observa a presença de retardo mental com níveis variando entre severo e profundo (Gillberg \& Coleman, 1996). Assim, atualmente, nenhum profissional qualificado propõe a existência de "uma causa" para o autismo. O máximo que se tem proposto é a identificação de fatores genéticos e biológicos que parecem contribuir para a exibição de certos sintomas ou características particulares, ou de subtipos específicos dentro da população geral de indivíduos autistas (e.g., Happé, Ronald, \& Plomin, 2006).

Várias pesquisas têm oferecido evidências que sugerem algum nível de participação de fatores genéticos nos TGDs. Porém, até o momento, não se sabe ao certo que genes 
específicos parecem contribuir para os fenótipos característicos dos TGDs nem como exatamente eles atuam, interagindo entre si e/ou com fatores ambientais específicos. Rich et al. (1999) comentam que pelo menos $10 \mathrm{a}$ 15 genes diferentes devem interagir para levar a um quadro de TGD. Grande número de estudos tem demonstrado que anormalidades genéticas nos cromossomos 7 e 15 (mais especificamente, na região $15 q 11-q 13$ ) resultam em fenótipos que variam de atrasos no desenvolvimento e na linguagem a autismo (e.g., Dykens, Sutcliffe, \& Levitt, 2004; Nurmi et al., 2003). Estudos mais recentes têm também apontado mutações no cromossomo 16, que, apesar de raras, foram 100 vezes mais prevalecentes em crianças com TGDs do que na população em geral (e.g., Weiss et al., 2008). Entretanto, esses resultados não são conclusivos, requerendo ainda que muitas outras investigações sejam realizadas.

Vale ressaltar também que recentemente tem havido uma crescente suspeita de que a vacina tríplice viral (usada na prevenção contra o sarampo, a caxumba e a rubéola) e/ou o Timerosol (um preservativo à base de mercúrio amplamente utilizado em vacinas) são possíveis causadores de autismo. Apesar de existir um grande grupo de pais e profissionais que defendem arduamente tal afirmação, até o momento, nenhum dos inúmeros estudos realizados em vários países, como Estados Unidos, Dinamarca, Japão, Canadá, entre outros, ofereceram evidências positivas nesse sentido (e.g., Center for Biologics Evaluation and Research [CBER], n.d.; Fombonne, 2008; Fombonne et al., 2006; Ip, Wong, Ho, Lee, \& Wong, 2004; Madsen et al., 2002, 2003; Parker, Schwartz, Todd, \& Pickering, 2004; Rutter, 2005; Strauss \& Bigham, 2001; Thompson et al., 2007; Verstraeten et al., 2003). De toda forma, desde 1999, foi formalmente requerido aos fabricantes de vacinas nos Estados Unidos, pelo Serviço de Saúde Pública, a eliminar ou a minimizar a quantidade de Timerosol das vacinas (American Academy of Pediatrics [AAP], 1999). Apesar de todos os esforços que estão sendo realizados nos Estados Unidos para reduzir ao mínimo a exposição geral da população ao mercúrio, a incidência dos casos de autismo continua aumentando no país (Newsom \& Hovanitz, 2006; Schechter \& Grether, 2008).

\section{Prevalência/incidência}

Como já destacado anteriormente, a incidência de casos de autismo tem aumentado de forma significativa durante as últimas décadas. Os primeiros estudos epidemiológicos indicavam uma prevalência de 4 a 5 casos de autismo infantil por 10.000 nascimentos (Lotter, 1966; Wing \& Gould, 1979). Investigações mais recentes, porém, estimam um aumento drástico de casos, atingindo a média de 40 e 60 casos a cada 10.000 nascimentos (e.g., Baird et al., 2000; Barbaresi et al.,2006; Bertrand et al., 2001; Chakrabarti \& Fombonne, 2001; Fombonne et al., 2006; Gernsbacher, Dawson, \& Goldsmith, 2005; Schechter \& Grether, 2008).

Apesar de muitas pesquisas e debates, até o momento, não se sabe ao certo se esse aumento realmente reflete um crescimento genuíno do número de casos. Contudo, mesmo que estejamos longe de encontrar uma resposta definitiva para tal pergunta, certamente podemos afirmar que esse aumento se deve, pelo menos em parte, a outros fatores, como a recente ampliação dos critérios diagnósticos, permitindo, assim, que maior gama de casos (com perfis desenvolvimentais mais variados) seja incluída dentro do espectro. Existe também uma melhora na capacitação dos profissionais, o que leva a melhor detecção de casos que 
antes não eram diagnosticados, ou eram diagnosticados erroneamente (e.g., Barbaresi et al., 2006; Coury \& Nash, 2003; Fombonne et al., 2006).

Quanto à proporção de incidência entre indivíduos do sexo masculino e feminino, estudos indicam que o autismo é quatro vezes mais prevalecente em meninos do que em meninas em indivíduos com níveis normais de inteligência (Newsom \& Hovanitz, 2006). Essa prevalência, porém, tende a diminuir significativamente quando se compara meninos e meninas autistas com níveis intelectuais mais comprometidos (retardo mental profundo), chegando a 1,3 casos de meninos para cada 1 caso de menina investigada (Yeargin-Allsopp et al., 2003). Isso sugere que, apesar de casos de autismo serem mais raros em meninas, estes tendem a ser acompanhados por maior comprometimento cognitivo e funcional.

\section{Considerações práticas durante o processo diagnóstico}

Apesar de o DSM-IV-TR fornecer os critérios básicos para a determinação do diagnóstico de autismo, em termos práticos, o processo diagnóstico não é tão simples quanto pode parecer à primeira vista. Além da grande diversidade de manifestação dos sintomas autísticos, existe também uma grande variedade em termos de quando a criança começa a exibir cada um dos diferentes sintomas, bem como em termos do perfil desenvolvimental de cada criança e das comorbidades que podem estar presentes em diferentes casos. Desse modo, os profissionais envolvidos no processo de diagnóstico precisam ser capazes de obter as informações necessárias de forma cuidadosa e de interpretar tais informações de forma criteriosa, de modo a determinar se os sintomas apresentados pela criança refletem adequadamente um quadro diagnóstico de autismo.

Um número cada vez maior de profissionais tem defendido que a forma mais adequada de se estabelecer o diagnóstico é de modo interdisciplinar, incluindo pelo menos um neuropediatra e um psicólogo com especialização em distúrbios do desenvolvimento. Esses profissionais têm a oportunidade de analisar cada caso conjuntamente, identificando as várias nuanças do quadro clínico da criança e oferecendo à família informações detalhadas não apenas acerca do diagnóstico mas também do perfil médico, cognitivo e adaptativo da criança. Além disso, esses profissionais devem orientar a família acerca das possibilidades de tratamentos e intervenções e encaminhála aos serviços e apoios necessários (e.g., Barbaresi et.al., 2006; Charman \& Baird, 2002; Filipek et al., 1999; Nash \& Coury, 2003; Newsom \& Hovanitz, 2006).

(1) Identificação de sintomas de risco: o primeiro passo para o diagnóstico precoce Uma vez que os sintomas de autismo começam a se manifestar bastante cedo na vida da criança (i.e., antes dos três anos), quanto mais cedo esses sintomas forem identificados, maiores as chances de a criança receber intervenções adequadas e exibir progressos desenvolvimentais mais significativos e duradouros. Por essa razão, faz-se essencial que mesmo os profissionais não especializados em diagnóstico de autismo, mas que trabalhem com a população infantil, sejam capazes de reconhecer os sintomas centrais. Uma vez que os sintomas de risco sejam identificados, a criança deve ser encaminhada para uma avaliação mais rigorosa, conduzida por uma equipe interdisciplinar especializada, para que um diagnóstico seja determinado. 
Déficits sociais, além de serem a marca central do autismo, são também os sintomas que se manifestam mais precocemente (até mesmo em crianças entre 12 e 18 meses de idade). Incluem, por exemplo, a falta ou o atraso em responder ao nome, aversão ao toque, dificuldade em estabelecer ou manter contato visual bem como em compartilhar interesses e estados emocionais com outros (atenção partilhada). Outros sintomas alarmantes nos dois primeiros anos de vida incluem a falta de balbucio aos 12 meses, de produção de palavras isoladas aos 16 meses e da combinação de duas palavras com a finalidade de se comunicar com outros aos 24 meses, bem como a ausência de brincadeiras de faz de conta aos 18 meses. Qualquer perda de habilidades verbais, comunicativas e/ou sociais em qualquer idade também se apresenta como um sintoma alarmante que requer avaliação (e.g., Barbaresi et.al., 2006; Charman \& Baird, 2002).

Assim, os profissionais da área infantil (pediatras, psicólogos, fonoaudiólogos, terapeutas ocupacionais, fisioterapeutas etc.) devem estar sempre alertas para a identificação desses sintomas de risco. Um número cada vez maior de pediatras nos Estados Unidos tem incluído instrumentos de avaliação de sintomas de risco para autismo como parte dos exames de rotina realizados nos seus consultórios. O Modified Checklist for Autism in Toddlers - M-CHAT (Lista Modificada de Verificação de Autismo em Crianças Pequenas, Robins, Fein, Barton, \& Green, 2001) tem sido o instrumento mais comumente utilizado em crianças a partir dos 18 meses de idade. Quando a criança se apresenta como de alto risco, os pediatras a encaminham para um processo de avaliação mais minucioso por uma equipe especializada.
(2) Componentes básicos necessários para uma avaliação diagnóstica adequada

A avaliação de diagnóstico deve, idealmente, ser feita por uma equipe interdisciplinar. Entretanto, tal configuração nem sempre se apresenta como realidade em muitos locais que oferecem avaliações diagnósticas. Desse modo, mesmo que o diagnóstico seja feito individualmente por um neuropediatra ou psicólogo especializado na área, ainda assim se recomenda que tal profissional encaminhe a criança para outras especialidades relevantes ao caso, de forma a assegurar que todos os domínios nos quais ela possa apresentar problemas sejam devidamente examinados e tratados. Assim, independentemente da avaliação diagnóstica ser feita individualmente ou por uma equipe interdisciplinar, faz-se necessário que os seguintes componentes estejam presentes durante tal processo:

(a) Entrevista clínica inicial com os pais ou responsáveis: constitui um elemento fundamental no processo diagnóstico, não apenas por ser o cenário através dos quais informações essenciais para o diagnóstico são obtidas, mas também por servir como um guia para a determinação de que outros passos parecem necessários para o restante da avaliação (exames genéticos, de audição, de visão, de níveis de chumbo no organismo, de atividades cerebrais, etc.). Para ser informativa, ela deve cobrir tópicos básicos como:

\section{História social e familiar da criança:} dinâmica familiar, história de problemas mentais, médicos, de desenvolvimento, e/ou de aprendizado entre membros da família imediata e extensa, etc;

História médica da criança: história da gravidez, parto e primeira infância, incluindo questões sobre exposição a tóxicos ou 
agentes químicos durante a gravidez e complicações durante o parto, história de infecções de ouvido recorrentes, alergias, problemas significativos de saúde que requeressem cirurgias, hospitalizações e/ou uso de medicação;

\section{História do desenvolvimento da criança:}

quando a criança atingiu diferentes marcos do desenvolvimento (i.e., quando começou a balbuciar, produzir as primeiras palavras, engatinhar, andar etc.), nível atual de atuação da criança em áreas diversas (habilidades comunicativas, sociais, motoras, pré-acadêmicas, cuidados pessoais, etc.), problemas de comportamento e de desenvolvimento (problemas com hábitos alimentares e de sono, comportamentos agressivos e autodestrutivos, comportamentos repetitivos, ritualísticos e estereotipados, irritabilidade, hipersensibilidade a certos estímulos sensoriais, hiperatividade, problemas de atenção).

\section{Instrumentos auxiliares no diagnóstico:}

existem atualmente vários instrumentos que auxiliam na determinação de sintomas de autismo durante o processo de avaliação diagnóstica. Profissionais em vários países têm se utilizado de uma combinação de diferentes instrumentos, analisando todas as informações obtidas através destes, juntamente às informações obtidas através da entrevista inicial com os pais bem como da observação direta da criança em diferentes contextos. Dessa forma, é realizada uma determinação acerca do diagnóstico. Vale destacar que os resultados oferecidos por qualquer um desses instrumentos não devem ser utilizados per se, como determinantes de um diagnóstico.

Dentre os instrumentos diagnósticos em formato de questionário para os pais/ professores, podemos citar o Pervasive
Developmental Disorder Behavior Inventory - PDDBI (Inventário Comportamental dos Transtornos Invasivos do Desenvolvimento, Cohen \& Sudhalter, 2005) e o Gilliam Autism Rating Scale - Second Edition - GARS-2 (Escala de Avaliação de Autismo de Gilliam - 2a ed.; Gilliam, 2006). Existe também o Childhood Autism Rating Scale - CARS (Escala de Avaliação de Autismo na Infância, Schopler, Reichler, \& Renner, 1988), no qual os comportamentos da criança são avaliados de acordo com as informações obtidas através de entrevistas com os pais e de observações diretas da criança. Finalmente, um número cada vez maior de profissionais tem se utilizado do Autism Diagnostic Interview - Revised - ADI-R (Entrevista Diagnóstica para Autismo - revisado, Lord, Rutter, \& Le Couteur, 1994) e do Autism Diagnostic Observation Schedule - ADOS (Observação Diagnóstica Programática para Autismo, Lord, Rutter, Dilavore, \& Risi, 1999). Esses dois instrumentos foram elaborados para serem utilizados de forma complementar. Enquanto o ADI-R corresponde a uma entrevista semiestruturada com os pais, o ADOS corresponde a um programa semi-estruturado de atividades e entrevistas realizadas diretamente com a criança.

Avaliação médica: como já discutido anteriormente, apesar de o diagnóstico de autismo ser realizado com base em critérios comportamentais, ainda assim avaliações médicas são bastante necessárias, tanto como parte do diagnóstico diferencial quanto da investigação de comorbidades. Fatores médicos usualmente investigados incluem distúrbios de ordem neurológica (como epilepsia, convulsões, esclerose tuberosa), metabólica (como erros inatos em aminoácido, carboidrato, peptídios, metabolismo mitocondrial, intoxicação por chumbo) e genética (como a síndrome do X frágil e outras mutações genéticas, 
especialmente nos cromossomos 7 e 15) (e.g., Barbaresi et al., 2006; Curatolo, Porfirio, Manzi, \& Seri, 2004;. Filipek et al., 1999; Nash \& Coury, 2003). Além disso, outras possíveis condições e alterações são também investigadas, como problemas sensoriais (visão, audição), problemas particulares de linguagem (dispraxia verbal, disartria, apraxia) bem como dificuldades alimentares e de sono. Uma investigação médica aprofundada é essencial, especialmente no caso de crianças que apresentam fatores de risco para problemas de ordem médica, como: (1) disformias faciais específicas e/ou outras anomalias craniofaciais e corporais, (2) história familiar positiva para desordens neurológicas, genéticas, metabólicas ou desenvolvimentais e/ou (3) história de complicações médicas pré, peri e pós-natal (e.g., Barbaresi et al., 2006; Nash \& Coury, 2003).

Avaliação psicológica: uma boa avaliação psicológica talvez seja um dos elementos mais úteis durante o processo diagnóstico, uma vez que fornecerá informações detalhadas acerca do funcionamento cognitivo e adaptativo da criança, o que é essencial para a formulação de um plano de intervenção individualizado. Como já mencionado anteriormente, crianças com autismo apresentam perfis bem diversos em termos de habilidades cognitivas, adaptativas, sociais, comunicativas e préacadêmicas, bem como na manifestação de variados padrões de estereotipias e problemas de comportamento. Desse modo, faz-se necessário que mensurações diretas do funcionamento da criança sejam obtidas para que se possa determinar que habilidades específicas ela apresenta (suas competências) e em que áreas de funcionamento 4exibe dificuldades (seus déficits).

Dependendo da faixa etária da criança, diferentes testes têm sido utilizados como parte de avaliações psicológicas realizadas por profissionais nos Estados Unidos. Para crianças mais novas, são geralmente administrados testes como o Mullen Scales of Early Learning (Escalas de Aprendizado no Início da Vida, de Mullen (Mullen, 1995) e o Bayley Scales of Infant and Toddler Development (3rd ed.), Bayley-III (Escalas do Desenvolvimento Infantil, de Bayley [3a ed.]), (Bayley, 2006), que oferecem uma estimativa da atuação da criança em diferentes áreas do desenvolvimento, como processamento de informações visuais, habilidades motoras refinadas e grosseiras e linguagem receptiva e expressiva. Mensurações a partir de entrevistas realizadas com os pais ou os responsáveis pela criança também podem ser obtidas com o uso de instrumentos como o Developmental Profile (3rd ed.) DP-3 (Perfil de Desenvolvimento [3a ed.] Alpern, Boll, \& Shearer, 1980). O uso do DP-3 é recomendado especialmente nos casos em que a avaliação direta da criança foi problemática ou impossível de ser realizada.

Para crianças mais velhas (mais ou menos a partir dos quatro anos de idade), são usualmente administrados testes de inteligência como o Stanford-Binet (5th ed.) (Stanford-Binet [5a ed.], Roid, 2003) ou o Wechsler Intelligence Scale for Children (4th ed.) WISC-IV (Escala de Inteligência para Crianças de Wechsler [4a ed.]), Wechsler, 2003), os quais oferecem uma estimativa do funcionamento cognitivo geral da criança. Devido ao fato de a maioria das crianças autistas possuir dificuldades marcantes com a compreensão e o uso de linguagem verbal, tais crianças podem ter seu desempenho nos testes pelo menos parcialmente afetado pela sua inabilidade em compreender as instruções do examinador. Esse tipo de problema tem sido geralmente compensado com a utilização de testes não-verbais, como o Leiter Reviewed - Leiter-R (Leiter - Revisado, Roid \& Miller, 2002) em conjunto com os demais 
testes. $\mathrm{O}$ Leiter-R não requer qualquer uso de habilidades verbais por parte da criança, uma vez que as instruções são fornecidas através de gestos e mímicas e as respostas não envolvem nenhum uso de linguagem. Dessa forma, pode ser obtida uma estimativa do funcionamento cognitivo não-verbal da criança, que fornece uma idéia acerca do quanto a criança está aprendendo a partir da interação com o seu ambiente através de meios não-verbais.

Além disso, mensurações das habilidades adaptativas da criança (como atuação independente da criança em áreas diversas, incluindo habilidades físicas, comunicativas, sociais, de cuidados pessoais, domésticas, comunitárias e vocacionais) também constituem parte integrante de avaliações psicológicas em crianças autistas. Essa mensuração é feita através do uso de instrumentos em formato de entrevistas ou de questionários para os pais ou responsáveis pela criança. Instrumentos como o Scales of Independent Behavior - Revised - SIB-R (Escalas de Comportamento Independente, Bruininks, Woodcock, Weatherman, \& Hill, 1996) e o Vineland Adaptive Behavior Scales (2nd ed.) (Escalas de Comportamento Adaptativo de Vineland [2a ed.]), Sparrow, Cicchetti, \& Balla, 2005) são geralmente utilizados para tal finalidade.

A obtenção de estimativas das habilidades cognitivas e adaptativas da criança se apresenta como de particular valor informativo durante o processo de avaliação, afinal, são os dois elementos básicos utilizados para o estabelecimento de um diagnóstico de retardo mental (e.g., American Association on Mental Retardation [AAMR], 2006; Jacobson \& Mulick, 1996). Vale a pena esclarecer, entretanto, que um diagnóstico de retardo mental significa que a criança está se desenvolvendo e aprendendo através do seu meio ambiente muito mais lentamente do que a maioria das crianças na mesma faixa etária. Isso, porém, não necessariamente constitui uma condição crônica e inalterável.

Em muitos casos, por exemplo, quando a criança é diagnosticada em idade precoce e recebe intervenções adequadas, ela pode eventualmente atingir níveis de desenvolvimento e aprendizado mais apropriados para a sua faixa etária e não mais recebe um diagnóstico de retardo mental, ou seja, a implementação de intervenções pode, em alguns casos, acelerar a velocidade de aprendizado e desenvolvimento da criança a tal ponto que ela venha a atingir níveis apropriados para a sua faixa etária. Mesmo quando as crianças não chegam a exibir uma aceleração da velocidade de aprendizado e desenvolvimento suficiente para não mais receber um diagnóstico de retardo mental, elas podem, ainda assim, passar a apresentar níveis mais leves de retardo mental (Lovaas, Koegel, Simmons, \& Long, 1973; Lovaas, 1987; Smith, Eikeseth, Klevstrand, \& Lovaas, 1997). Desse modo, o retardo mental não é considerado um estado necessariamente permanente da criança, mas sim, uma representação do padrão de desenvolvimento e aprendizado da criança durante um determinado período de sua vida.

Quando o retardo mental e os sintomas autísticos são mais severos e associados a alguma condição médica (e.g., microcefalia, epilepsia, traumas cerebrais, síndromes genéticas, etc.), geralmente, nesses casos, os ganhos desenvolvimentais e funcionais através de intervenções são bem mais modestos, porém, podem ser, ainda assim, significativos e progressivos (e.g., Nash \& Coury, 2003). Por isso, mesmo nesses casos, intervenções intensivas e individualizadas são altamente recomendadas. Tais intervenções são reconhecidas por promoverem 
progressos sucessivos e relevantes em termos desenvolvimentais e comportamentais em crianças com qualquer perfil desenvolvimental, contribuindo para que estas se tornem participantes os mais ativos possível no seu meio familiar e social, sendo capazes de freqüentar espaços educacionais, profissionais e sociais que lhes forneçam os serviços e apoios necessários (e.g., Barbaresi et al., 2006).

\section{Encaminhamento para outros profissionais e para intervenções apropriadas: uma} vez que o diagnóstico de autismo tenha sido confirmado ou desconfirmado, os profissionais precisam, então, determinar se algum encaminhamento ainda se faz necessário. Isso inclui encaminhamentos para terapeutas ocupacionais, fisioterapeutas, fonoaudiólogos, neurologistas, geneticistas e/ou para profissionais que lidam com problemas alimentares e/ou de sono. É essencial, também, que a criança e a sua família sejam encaminhadas para programas educacionais específicos, como os programas de intervenção comportamental intensiva e para programas de treinamento de pais voltados para a eliminação de problemas de comportamento da criança. Desse modo, pode-se assegurar que todas as diferentes áreas em que a criança apresente problemas serão efetivamente investigadas e tratadas. Avaliações psicológicas anuais são também altamente recomendadas, para que se possa monitorar o progresso da criança ao longo dos anos e auxiliar na revisão dos programas e intervenções recebidos por ela.

\section{Comentários finais}

Através das informações discutidas neste artigo, esperamos ter contribuído para o esclarecimento de, pelo menos, alguns aspectos centrais acerca do transtorno autista. Apesar de as pesquisas ainda não terem avançado o suficiente para se determinar fatores e processos específicos que estejam definitivamente envolvidos na etiologia do autismo, acreditamos que já avançamos bastante em termos de informações que dão suporte à implementação de práticas diagnósticas adequadas e de boa qualidade. A identificação de sintomas de risco como parte da rotina de profissionais que trabalham com a população infantil, bem como a formação de equipes interdisciplinares especializadas em diagnóstico de autismo, por exemplo, têm permitido que o diagnóstico seja determinado de forma bastante segura, mesmo em crianças de tenra idade. Isso constitui um avanço de importância primordial, visto que a idade na qual a criança começa a receber intervenções apropriadas representa um dos elementos essenciais para um melhor prognóstico em termos de seu desenvolvimentos e de sua atuação (e.g., Charman \& Baird, 2002).

Nosso contato com profissionais que trabalham na área prática em vários Estados no Brasil nos faz acreditar que as práticas diagnósticas de autismo atualmente implementadas em muitas partes do País ainda carecem de melhores diretrizes e organização geral para se tornarem mais eficazes. Nesse sentido, os pontos apresentados neste artigo são por nós avaliados como a ilustração de um modelo de prática eficiente já implementado em vários países, sendo, portanto, essenciais para instigar os profissionais aqui no Brasil a questionarem alguns apectos das práticas atuais em vigência em boa parte do País. Com isso, esperamos estimular a elaboração de modelos norteadores mais eficientes e adequados tanto no que se refere às práticas diagnósticas quanto à implementação de sistemas de apoio e intervenção para os indivíduos com autismo e suas famílias no Brasil.

Apesar de os pontos discutidos neste artigo darem ênfase a questões práticas, também 
esperamos que as problemáticas aqui levantadas gerem reflexões e questionamentos no âmbito acadêmico e de pesquisa científica. Acreditamos ser de suma importância que discussões sobre tais problemáticas se tornem parte integrante da formação de profissionais não apenas na Psicologia mas também em áreas da saúde em geral e em outras áreas afins, especialmente naquelas que tenham como alvo a população infantil. Aspectos como a relevância de se desenvolver trabalhos de natureza interdisciplinar, bem como as vantagens de se estabelecer um diagnóstico precoce, por exemplo, parecem ser tópicos ainda hoje polêmicos entre membros da academia. Por isso, discussões sobre essas temáticas se fazem essenciais para a formação de profissionais críticos e qualificados. Além disso, tais problemáticas podem também ser de grande contribuição para o desenvolvimento de novas linhas de pesquisa, com o intuito de preencher algumas das inúmeras lacunas ainda existentes no conhecimento atual acerca do autismo.

Micheline Silva*

Doutora em Psicologia do Desenvolvimento pela Clark University - EUA.

Universidade de Brasília - Instituto de Psicologia.

James A. Mulick

Ph.D, Nationwide Children's Hospital - Autism Center \& Developmental Assessment Program Columbus, Ohio, EUA.

*Endereço para envio de correspondência:

Universidade de Brasília, Instituto de Psicologia - PED - LabMis - ICC Asa Norte. Cep: 70.910-900 - Brasília, DF E-mail: msilva@wowway.com

Recebido 09/05/2008; Reformulado 22/08/2008; Aprovado 30/08/2008. 


\section{Referências}

Alpern, G., Boll, T., \& Shearer, M. (1980). Developmental Profile III. Aspen: Psychological Development Publications.

American Academy of Pediatrics. (1999). Joint statement of the American Academy of Pediatrics (AAP) and the United States Public Health Service (USPHS) (RE9937). Pediatrics, $104,568-569$.

American Association on Mental Retardation. (2006). Retardo mental: definição, classificação e sistemas de apoio (10a ed.). Porto Alegre: Artmed.

American Psychiatric Association. (2003). DSM-IV-TR, manual diagnóstico e estatístico de transtornos mentais (4a ed. rev.) Porto Alegre: Artmed.

Bailey, A., Philips, W. \& Rutter, M. (1996). Autism: Towards an integration of clinical, genetic, neuropsychological, and neurobiological perspectives. Journal of Child Psychology and Psychiatry, 37, 89-126.

Baird, G., Charman, T., Baron-Cohen, S., Cox, A., Swettenham, J., Wheelwright, S., et al. (2000). A screening instrument for autism at 18 months of age: A 6-year follow-up study. Journal of the American Academy of Child and Adolescent Psychiatry, 39, 694-702.

Barbaresi, W. J., Katusic, S. K., Colligan, R. C., Weaver, A. L., \& Jacobsen, S. J. (2005). The incidence of autism in Olmsted County, Minnesota, 1976-1997: Results from a populationbased study. Archive of Pediatric and Adolescent Medicine, 159, 37-44

Barbaresi, W. J., Katusic, S. K., \& Voigt, R. G. (2006). Autism A review of the state of the science for pediatric primary health care clinicians. Archive of Pediatric and Adolescent Medicine, 160, 1167-1175.

Bayley, N. (2006). Bayley scales of infant and toddler development (3rd ed.). San Antonio, TX: Psych Corp - Harcourt Assessment.

Bertrand, J., Mars, A., Boyle, C., Bove, F., Yeargin-Allsopp, M., \& Decoufle, P. (2001). Prevalence of autism in a United States population: The Brick Township, New Jersey, investigation. Pediatrics, 108, 1155-1161.

Brambilla, P., Hardan, A., Ucelli Di Nemi, S., Perez, J., Soares, J. C., \& Barale, F. (2003). Brain anatomy and development in autism: Review of structural MRI studies. Brain Research Bulletin, 61, 557-569.

Bruininks, R. H., Woodcock, R. W., Weatherman, R. F., \& Hill, B. K. (1996). Scales of Independent Behavior (ed. rev.). Itasca, IL: Riverside.

Center for Biologics Evaluation and Research - US Food and Drug Administration. Department of Health and Human Services. (n.d.). Thimerosal in vaccines. Recuperado em 08 de janeiro de 2008, de http://www.fda.gov/CBER/vaccine/ thimerosal.htm

Chakrabarti, S., \& Fombonne, E. (2001). Pervasive developmental disorders in preschool children. Journal of the American Medical Association, 285, 3093-3099.

Chakrabarti, S., \& Fombonne, E. (2005). Pervasive developmental disorders in preschool children: Confirmation of high prevalence. American Journal of Psychiatry, 126, 133-1141.

Challman, T. D., Barbaresi, W. J., Katusic, S. K., \& Weaver, A (2003). The yield of the medical evaluation of children with pervasive developmental disorders. Journal of Autism and Developmental Disorders, 33, 187-192.

Charman, T., \& Baird, G. (2002). Practitioner review: Diagnosis of autism spectrum disorder in 2- and 3-year-old children. Journal of Child Psychology and Psychiatry, 43(3), 289-305.

Cohen, I. L., \& Sudhalter, V. (2005). Pervasive Developmental Disorder Behavior Inventory. Lutz, FL: Psychological Assessment Resources.
Courchesne, E., Caper, R., \& Akshoomoff, N. (2003). Evidence of brain overgrowth in the first year of life in autism. Journal of the American Medical Association, 290, 337-344.

Coury, D. L., \& Nash, P. L. (2003). Epidemiology and etiology of autistic spectrum disorders difficult to determine. Pediatric Annals, 32(10), 696-700.

Curatolo, P., Porfirio, M. C., Manzi, B., \& Seri, S. (2004). Autism in tuberous sclerosis. European Journal of Pediatric Neurology, 8, 327-332.

Dykens, E. M. Sutcliffe, J. S , \& Levitt, P (2004). Autism and 15Q11-Q13 disorders: Behavioral, genetic, and pathophysiological issues. Mental Retardation and Developmental Disabilities Research Reviews, 10, 284-291.

Filipek, P. A., Accardo, P. J., Baranek, G. T., Cook, E. H., Dawson, G., Gordon, B., et al. (1999). The screening diagnosis of Autistic Spectrum Disorders. Journal of Autism and Developmental Disorders, 29(6), 439-484.

Fombonne, E. (2008). Thimerosal disappears but autism remains Archive of General Psychiatry, 65, 15-16.

Fombonne, E., Zakarian, R., Bennett, A., Meng, L., \& McleanHeywood, D. (2006). Pervasive developmental disorders in Montreal, Quebec, Canada: Prevalence and links with immunizations. Pediatrics, 118(1), e139-e150.

Gernsbacher, M., Dawson, M., \& Goldsmith, H. (2005). Three reasons not to believe in an autism epidemic. Current Directions in Psychological Science, 14, 55-58.

Gillberg, C., \& Coleman, M. (1996). Autism and medical disorders: A review of the literature. Developmental Medicine and Child Neurology, 38, 191-202.

Gilliam, J. E. (2006). Gilliam autism rating scale (2nd ed.). Austin TX: Pro-ed.

Goldberg, W. A., Osann, K., Filipek, P. A., Laulhere, T., Jarvis, K Modahl, C., et al. (2003). Language and other regression: Assessment and timing. Journal of Autism and Developmental Disorders, 33, 607-616.

Happé, F., Ronald, A., \& Plomin, R. (2006). Time to give up on a single explanation for autism. Nature Neuroscience, 9, 1218-1220.

Ip, P., Wong, V., Ho, M., Lee, J., \& Wong, W. (2004). Mercury exposure in children with autistic spectrum disorder: Casecontrol study. Journal of Child Neurology, 19, 431-434.

Jacobson, J. W., \& Mulick, J. A. (1996). Manual of diagnosis and professional practice in mental retardation. Washington, DC: APA.

Lindsay, R. L., \& Aman, M. G. (2003). Pharmacologic therapies aid treatment for autism. Pediatric Annals, 32(10), 671-676.

Lord, C., Rutter, M., Dilavore, P., \& Risi, S. (1999). Autism diagnostic observation schedule. Los Angeles: Western Psychological Services.

Lord, C., Rutter, M., \& Le Couteur, A. (1994). Autism diagnostic interview revised: A revised version of a diagnostic interview for caregivers of individuals with possible pervasive developmental disorders. Journal of Autism and Developmental Disorders, 24(5), 659-685.

Lord, C., Shulman, C., \& Dilavore, P. (2004). Regression and word loss in autistic spectrum disorders. Journal of Child Psychology and Psychiatry, 45, 936-955.

Lotter, V. (1966). Epidemiology of autistic conditions in young children. I. Prevalence. Social Psychiatry, 1, 124-137.

Lovaas, O. I. (1987). Behavior treatment and normal education and intellectual functioning in young autistic children. Journal of Consulting and Clinical Psychology, 55, 3-9. 
Lovaas, O. I., Koegel, R., Simmons, J. Q., \& Long, J. S. (1973). Some generalization and follow-up measures on autistic children in behavior therapy. Journal of Applied Behavior Analysis, 6, 131-166.

Madsen, K. M., Hviid, A., Vestergaard, M., Schendel, D., Wohlfahrt, J., Thorsen, P., et al. (2002). A population-based study of measles, mumps, and rubella vaccination and autism. New England Journal of Medicine, 347, 1477-1482.

Madsen, K. M., Lauritsen, M. P., Pedersen, C. B., Thorsen, P., Plesner, A. M., Andersen, P. H., et al. (2003). Thimerosal and the occurrence of autism: Negative ecological evidence from Danish population-based data. Pediatrics, 112, 604-606.

Mercadante, M. T., Gaag, R. J. V., \& Schwartzman, J. S. (2006). Transtornos invasivos do desenvolvimento não-autísticos: Sindrome de Rett, transtorno desintegrativo da infância e transtornos invasivos do desenvolvimento sem outra especificação. Revista Brasileira de Psiquiatria, 28(supl. I), S12-S20.

Mullen, E. (1995). Infant Mullen scales of early learning. Circle Pines, MN: American Guidance Service.

Muller, R., Kleinhans, N., Kemmotsu, N., Pierce, K., \& Courchesne, E. (2003). Abnormal variability and distribution of functional maps of autism: An MRI study of visuomotor learning. American Journal of Psychiatry, 160, 1847-1862.

Mundy, P. (2003). Annotation: The neural basis of social impairments in autism: The role of the dorsal medial-frontal cortex and anterior cingulate system. Journal of Child Psychology and Psychiatry, 44, 793-809.

Nash, P. L., \& Coury, D. L. (2003). Screening tools assist with diagnosis of autism spectrum disorders. Pediatric Annals, 32(10), 664-670.

Newsom, C., \& Hovanitiz, C. A. (2006). Autistic spectrum disorders. In E. J. Mash, \& R. A. Barkley (Eds.), Treatment of childhood disorders (3rd ed., pp. 455-511). New York: Guilford Press.

Nurmi, E. L., Amin, T., Olson, L. M., Jacobs, M. M., McCauley, J. L., Lam, A. Y., et al. (2003). Dense linkage disequilibrium mapping in the 15q11-q13 maternal expression domain yields evidence for association in autism. Molecular Psychiatry, 8, 624-634.

Parker, S. K., Schwartz, B., Todd, J., \& Pickering, L. K. (2004). Thimerosal-containing vaccines and autistic spectrum disorder: A critical review of published original data. Pediatrics, $114,793-804$

Redcay, E., \& Courchesne, E. (2005). When is the brain enlarged in autism? A meta-analysis of all brain size reports. Biological Psychiatry, 58, 1-9.

Robins, D. L., Fein, D., Barton, M. L., \& Green, J. A. (2001). The modified checklist for autism in toddlers: An initial study investigating the early detection of autism and pervasive developmental disorders. Journal of Autism and Developmental Disorders, 31(2), 131-144.

Roid, G. H. (2003). Stanford-Binet intelligence scale (5th ed.). Itasca, IL: Riverside.

Roid, G. H., \& Miller, L. J. (2002). Leiter international performance scale (ed. rev.). Wood Dale, IL: Stoelting.

Rutter, M. (2005). Autism research: Lessons from the past and prospects for the future. Journal of Autism and Developmental Disorders, 35, 241-257.

Schechter, R., \& Grether, J. K. (2008). Continuing increases in autism reported to California's Developmental Services
System: Mercury in retrograde. Archive of General Psychiatry, 65(1), 19-24.

Schopler, E., Reichler, R. J., \& Renner, B. R. (1988). The childhood autism rating scale. Los Angeles, CA: Western Psychological Services.

Schreck, K. A., \& Mulick, J. A. (2000). Parental report of sleep problems in children with autism. Journal of Autism and Developmental Disorders, 30, 127-135.

Shannon, M., \& Graef, J. (1997). Lead intoxication in children with pervasive developmental disorders. Journal of Toxicology - Clinical Toxicology, 34, 177-182.

Siperstein, R., \& Volkmar, F. (2004). Brief report: Parental reporting of regression in children with pervasive developmental disorders. Journal of Autism and Developmental Disorders, $34,731-734$

Smith, T., Eikeseth, S., Klevstrand, M., \& Lovaas, O. I. (1997). Intensive behavior treatment for preschoolers with severe mental retardation and pervasive developmental disorder. American Journal of Mental Retardation, 102, 238-249.

Sparrow, S. S., Cicchetti, D. V., \& Balla, D. A. (2005). Vineland adaptive behavior scales (2nd ed.). Circle Pines, MN: American Guidance Service.

Strauss, B., \& Bigham, M. (2001). Does measles-mumps-rubella (MMR) vaccination cause inflammatory bowel disease and autism? Canada Communicable Disease Report, 27, 65-72.

Thompson, W. W., Price, C., Goodson, B., Shay, D. K., Benson, P., Hinrichsen, V. L., et al. (2007). Vaccine safety datalink team: Early thimerosal exposure and neuropsychological outcomes at 7 to 10 years. New England Journal of Medicine, 357(13), 1281-1292.

Verstraeten, T., Davis, R. L., Destefano, F., Lieu, T. A., Rhodes, P. H., Black, S. B., et al. (2003). Safety of thimerosal-containing vaccines: A two-phased study of computerized health maintenance organization databases. Pediatrics, 112, 1039-1048.

Wechsler, D. (2003). Wechsler intelligence scale for children (4th ed.). San Antonio, TX: The Psych Corp - Harcourt Assessment Company.

Weiss, L. A., Shen, Y., Korn, J. M., Arking, D. E., Miller, D. T., Fossdal, R., et al. (2008). The autism consortium: Association between microdeletion and microduplication at 16p11.2 and autism. New England Journal of Medicine, 358(7), 667-675.

Werner, E., \& Dawson, G. (2005). Validation of the phenomenon of autistic regression using home videotapes. Archives of General Psychiatry, 62, 889-895.

Wing, L., \& Gould, J. (1979). Severe impairments of social interaction and associated abnormalities in children: Epidemiology and classification. Journal of Autism and Developmental Disorders, 9, 11-29.

World Health Organization. (1992). Classification of mental and behavioral disorders: Clinical descriptions and diagnostic guidelines. Geneva.

Yeargin-Allsopp, M., Rice, C., Karapurkar, T., Doernberg, N., Boyle, C., \& Murphy, C. (2003). The prevalence of autism: Metropolitan Atlanta. The Journal of the American Medical Association, 289, 49-55.

Zwaigenbaum, L., Bryson, S., Rogers, T., Roberts, W., Brian, J. \& Szatmari, P. (2005). Behavioral manifestations of autism in the first year of life. International Journal of Developmental Neuroscience, 23, 143-152. 